\title{
On Bilevel Variational Inequalities
}

\author{
Zhongping Wan · Jia-wei Chen
}

Received: 13 May 2013 / Revised: 15 November 2013 / Accepted: 7 December 2013 /

Published online: 18 December 2013

(C) Operations Research Society of China, Periodicals Agency of Shanghai University, and

Springer-Verlag Berlin Heidelberg 2013

\begin{abstract}
A class of bilevel variational inequalities (shortly (BVI)) with hierarchical nesting structure is firstly introduced and investigated. The relationship between (BVI) and some existing bilevel problems are presented. Subsequently, the existence of solution and the behavior of solution sets to (BVI) and the lower level variational inequality are discussed without coercivity. By using the penalty method, we transform (BVI) into one-level variational inequality, and establish the equivalence between (BVI) and the one-level variational inequality. A new iterative algorithm to compute the approximate solutions of (BVI) is also suggested and analyzed. The convergence of the iterative sequence generated by the proposed algorithm is derived under some mild conditions. Finally, some relationships among (BVI), system of variational inequalities and vector variational inequalities are also given.
\end{abstract}

Keywords Bilevel variational inequalities $\cdot$ Bilevel programs $\cdot$ System of variational inequality $\cdot$ Vector variational inequality $\cdot$ Penalty method

Mathematics Subject Classification (2000) 90C33 · 49J40 - 47H05

This work was supported by the Natural Science Foundation of China (Nos. 71171150, 11201039), the Doctor Fund of Southwest University (No. SWU113037) and the Fundamental Research Funds for the Central Universities (No. XDJK2014C073).

\section{Z. Wan}

School of Mathematics and Statistics, Wuhan University, Wuhan, Hubei 430072, P.R. China e-mail: zpwan-whu@126.com

J.-w. Chen $(\varangle)$

School of Mathematics and Statistics, Southwest University, Chongqing 400715, P.R. China

e-mail: J.W.Chen713@163.com 


\section{Introduction}

Variational inequalities, which were introduced by Stampacchia [51] in 1964, have been intensively studied and widely applied to some practical problems arising in economics, transportation, network and structural analysis, elasticity, engineering and mechanics, supply chain management, finance and game theory (see, e.g., [10-12, 28, 33-36, 38, 39, 47, 49, 54, 55, 58, 61] etc.). Recently, optimization problem with variational inequality, equilibrium and complementarity constraints, have caused many authors' interests (see, e.g., [7, 18, 31, 32, 40-43, 45, 50, 56, 59, 60] etc.). Mordukhovich [44] studied an equilibrium problems with equilibrium constraints (EPECs) and mathematical programs with equilibrium constraints (MPECs) via multiobjective optimization, and obtained some optimality conditions for (EPECs) based on advanced generalized differential tools of variational analysis. Outrata [48] applied the (EPECs) to an oligopolistic market with several large and several small firms, derived two types of necessary conditions for a solution of this game and briefly discussed the possibilities of its computation. Su [52] defined equilibrium problems with equilibrium constraints by finding an equilibrium point that solves several mathematical program with equilibrium constraints, and studied it in stationarity conditions and algorithms aspects. Cervinka [8] also studied a class of (EPECs) in which lower-level one was described by Nash equilibrium problem, and applied (EPECs) to Oligopolistic market problem, deregulated electricity market model and traffic equilibrium with private toll roads.

In 2010, Moudafi [46] introduced a class of bilevel monotone equilibrium problem (shortly (BEP)): find $x \in S_{F}$ such that

$$
H(x, y) \geqslant 0, \quad \forall y \in S_{F},
$$

where $S_{F}$ is the solution set of the equilibrium problem: find $y \in K$ such that

$$
F(y, z) \geqslant 0, \quad \forall z \in K,
$$

where $K$ is a nonempty closed convex subset of a Hilbert space, and $H, F: K \times K \rightarrow$ $\mathbb{R}$ are two functions. He pointed out that (BEP) is a generalization of optimization problems with equilibrium, variational inequalities, complementarity constraints. By using the proximal method, an iterative algorithm to compute approximate solution of the (BEP) and the weak convergence of the iterative sequence generated by the algorithm were suggested and derived, respectively. Dinh and Muu [27] further studied the (BEP) in finite dimensional Euclidean space, and applied penalty function to converted (BEP) into one-level equilibrium problem.

Motivated by Moudafi's works [46], Ding [22, 23] considered the following bilevel mixed equilibrium problem (shortly (BMEP)) (1.1)-(1.2) in reflexive Banach spaces: find $x \in S_{f, \psi}$ such that

$$
h(x, y)+\phi(y, x)-\phi(x, x) \geqslant 0, \quad \forall y \in S_{f, \psi},
$$

where $S_{f, \psi}$ is the solution set of the mixed equilibrium problem: find $y \in K$ such that

$$
f(y, z)+\psi(z, y)-\psi(y, y) \geqslant 0, \quad \forall z \in K,
$$


where $E$ is a real Banach space with its dual space $E^{*}$, the norm and the dual pair between $E$ and $E^{*}$ are denoted by $\|\cdot\|$ and $\langle\cdot, \cdot\rangle$, respectively. Let $K$ be a nonempty closed and convex subset of $E, h, f: K \times K \rightarrow \mathbb{R} \cup\{+\infty\}$ and $\phi, \psi: E \times E \rightarrow$ $\mathbb{R} \cup\{+\infty\}$ are bifunctions. Ding [22, 23] studied the existence of solution and iterative algorithms for (BMEP) (1.1)-(1.2). Thereafter, Ding [24, 25], and Ding, Liou and Yao [26] further generalized the (BMEP) (1.1)-(1.2) in different ways. Inspired by Ding [22] and Moudafi [46], Chadli, Mahdioui and Yao [9] studied the following (BMEP) in reflexive Banach spaces: find $x \in S_{f, \psi}$ such that

$$
h(x, y) \geqslant 0, \quad \forall y \in S_{f, \psi},
$$

where $S_{f, \psi}$ is the solution set of mixed equilibrium problem (1.2). They derived some existence results of the bilevel model, constructed an iterative algorithm to approximate its solution by regularization method, and proved the convergence of the sequence generated by the proposed algorithm. Very recently, Anh, Khanh and Van [1] investigated the well-posedness for (BEP) and optimization problems with equilibrium constraints under the relaxed level closedness and pseudocontinuity assumptions, and gave some sufficient conditions and metric characterizations for wellposedness of (BEP). Anh, Kim and Muu [2] studied the following bilevel variational inequalities in finite dimensional Euclidean space: find $x^{*} \in \operatorname{Sol}(G, C)$ such that

$$
\left\langle F\left(x^{*}\right), x-x^{*}\right\rangle \geqslant 0, \quad \forall x \in \operatorname{Sol}(G, C),
$$

where $F: \operatorname{Sol}(G, C) \rightarrow \mathbb{R}^{n}, G: C \rightarrow \mathbb{R}^{n}, \operatorname{Sol}(G, C)$ is the set of solution of the variational inequality: find $y^{*} \in C$ such that

$$
\left\langle G\left(y^{*}\right), y-y^{*}\right\rangle \geqslant 0, \quad \forall y \in C .
$$

They used the extragradient algorithm to approximate the solution of the (BVI), and proved the sequence generated by the proposed algorithm converges to some solution of the problem. Clearly, the above bilevel variational inequalities is a special case of (BEP) in Moudafi [46].

We observe that all bilevel models of $[1,2,9,13,14,22-27,46]$ have no hierarchical nesting structure. Since the feasible region of the upper-level variational problem is defined by the solution set of the lower-level one, but the solution set of the lower-level one is not effected by the solution to the upper-level one.

On the other hand, bilevel programming (BP), which is a special program of mathematical optimization problems, is a hierarchical optimization problem where a subset of the variables is constrained to be a solution of a given optimization problem parameterized by the remaining variables. Namely, (BP) involves two optimization problems where the constraint region of the upper-level optimization problem known as the leader's problem is implicitly determined by the lower-level optimization problem known as the follower's problem parameterized in the decision variable of the leader. While the leader firstly makes his rational choice and the follower reacts optimally on the leader's selection, the leader's aim consists in finding such a selection which, together with the follower's response, minimizes some cost function (see, e.g., $[18,19]$ etc.). 
The general formulation of bilevel programming problem (BP) (see, e.g., $[15,18]$ etc.) is defined by

$$
\min _{x, y} F(x, y) \quad \text { subject to } x \in X, G(x, y) \leqslant 0, y \in S(x),
$$

where $S(x)$ is the set of solutions to the lower-level problem parameterized in $x$ :

$$
\min _{y} f(x, y) \quad \text { subject to } g(x, y) \leqslant 0,
$$

where $x \in \mathbb{R}^{n_{1}}$ and $y \in \mathbb{R}^{n_{2}}, F: \mathbb{R}^{n_{1}} \times \mathbb{R}^{n_{2}} \rightarrow \mathbb{R}$ and $f: \mathbb{R}^{n_{1}} \times \mathbb{R}^{n_{2}} \rightarrow \mathbb{R}$ are the upper-level and lower-level objective functions, respectively, while the vector-valued mappings $G: \mathbb{R}^{n_{1}} \times \mathbb{R}^{n_{2}} \rightarrow \mathbb{R}^{m_{1}}$ and $g: \mathbb{R}^{n_{1}} \times \mathbb{R}^{n_{2}} \rightarrow \mathbb{R}^{m_{2}}$ are the upper-level and lower-level constraints, respectively. For the past decades, (BP) has often been studied in various directions, and received increasing applications in many fields such as economics, electric utility, network design, transportation network, supply chain management, finance and game theory (see, e.g., [5, 15, 18, 19, 21, 53] etc.). So, it is a practical and useful tool for solving decision-making problems with hierarchical structure. In [20], Dempe and Dutta investigated the relationships between bilevel programming (BP) and mathematical program with complementarity constraints (MPCC), pointed out that (BP) is not a special case of (MPCC) even when the lower-level programming problem is a parametric convex optimization problem. In [57], Xu, Li and Yang discussed the so-called bilevel variational inequality (1.3)(1.4) by neural network method.

Find $(x, y)$ such that

$$
x \in \Omega_{2}, \quad\left(x^{\prime}-x\right)^{\mathrm{T}} y \geqslant 0 \quad \forall x^{\prime} \in \Omega_{2},
$$

where $x$ is a solution of the following variational inequality:

$$
x \in \Omega_{1}, \quad\left(x^{\prime}-x\right)^{\mathrm{T}}(f(x)-y) \geqslant 0 \quad \forall x^{\prime} \in \Omega_{1},
$$

where $\Omega_{1}, \Omega_{2}$ are two closed convex subset of $\mathbb{R}^{n}, \Omega_{2} \subset \Omega_{1}, f: \mathbb{R}^{n} \rightarrow \mathbb{R}^{n}$ is a vector-valued mapping, $y \in \mathbb{R}^{n}$ is a control variable. The bilevel problem (1.3)-(1.4) seems to have hierarchical nesting structure. Observe that the decision variables of the upper-level problem and the lower-level one are the same, the control variable $y \in \mathbb{R}^{n}$ is neither the decision variable of upper-level one nor that of lower-level one. Indeed, bilevel problem (1.3)-(1.4) can be viewed as two-stage problem parameterized in the control variable $y$.

The upper-level or lower-level problems are Nash equilibrium problems in the models of [44, 48, 52]. As we know, Nash equilibrium problem is a multiplayer games problem. In many practical problems, the upper-level or lower-level problems only involve one decision maker. Moreover, a Nash equilibrium problem can be equivalently reformulated as variational inequalities (see, e.g., [34, 37, 42] etc.). It is worth noting that the solution of (BP) must make the upper-level decision maker and the lower-level one achieve optimal condition. In many practical problems, we usually can only make a mutually acceptable solution (i.e., equilibrium solution) for the upper-level and the lower-level decision makers. The acceptable solution can be obtained by using variational inequality. 
Motivated and inspired by the above works, the aim of this paper is to introduce and investigate a general model of the bilevel variational inequalities (BVI) with hierarchical nesting structure that is analogous to (BP). The hierarchical process signifies that while the leader has the first choice and the follower responses optimally on the leader's selection, the leader's aim consists in finding such a selection which, together with the follower's response, is an optimal solution of the upper-level variational inequality. Subsequently, the existence of solution and the behavior of solution sets to (BVI) and the lower-level variational inequality are discussed under some suitable conditions. We apply the penalty method to transform (BVI) into one-level variational inequality, and establish the equivalence between (BVI) and the one-level variational inequality. A new iterative algorithm to compute the approximate solutions of (BVI) is suggested and analyzed. The strong convergence of the iterative sequence generated by the proposed algorithm is also proved under some mild conditions. Finally, some relationships among (BVI), system of variational inequalities and vector variational inequalities are presented.

The outline of the paper is as follows. We begin Sect. 2 by recalling the preliminary results and presenting the problem formulation of (BVI). In Sect. 3, we carry on the existence of solution and the behavior of solution sets to (BVI) and the lowerlevel variational inequalities. By using penalty method, we transform (BVI) into a one-level variational inequalities in Sect. 4. Based on Sect. 4, we construct an iterative algorithm to compute the approximation solution to (BVI), and analyze the convergence of the presented algorithm in Sect. 5. The relationships among (BVI), system of variational inequalities and vector variational inequalities are discussed in Sect. 6 .

\section{Preliminaries}

Throughout this paper, let $K$ be a nonempty subset of the $n$-dimensional Euclidean space $\mathbb{R}^{n}, \mathbb{R}_{+}^{m}$ denote the nonnegative orthant of $\mathbb{R}^{m}, \Phi: \mathbb{R}^{m} \times \mathbb{R}^{n} \times \mathbb{R}^{n} \rightarrow \mathbb{R} \cup\{+\infty\}$ and $\psi: \mathbb{R}^{m} \times \mathbb{R}^{m} \rightarrow \mathbb{R} \cup\{+\infty\}$ such that $\Phi(z, x, 0)=0$ and $\psi(z, 0)=0$ for all $z \in \mathbb{R}^{m}, x \in \mathbb{R}^{n}$, and let $T: K \rightarrow 2^{\mathbb{R}^{m}}$ be a set-valued mapping, where $2^{\mathbb{R}^{m}}$ is the family of all nonempty subsets of $\mathbb{R}^{m}$.

Consider the following bilevel variational inequalities (shortly (BVI)): find $(x, z) \in K \times \mathbb{R}^{m}$ such that

$$
\Phi(z, x, x-y) \leqslant 0, \quad \forall y \in K,
$$

where $z \in S(x), S(x)$ is the solution set of the following variational inequalities defined by bifunction with respect to a parameter $x$ : find $z \in T(x)$ such that

$$
\psi(z, z-v) \leqslant 0, \quad \forall v \in T(x) .
$$

Equations (2.1) and (2.2) are called the upper-level Stampacchia type variation inequality (shortly (USVI)) and the lower-level Stampacchia type variation inequality (shortly (LSVI)), respectively. The decision variables of problem (BVI) are divided into two classes, namely, the upper-level decision variable $x$ and the lower-level decision variable $z$. Denote the optimal solution set of (BVI) by $\Theta$. 
Next, we give some definitions similar to the terminology in bilevel programming [18] for the (BVI):

- The constraint region of (BVI):

$$
C=\{(y, v): \forall y \in K, v \in T(y)\} .
$$

- The projection of $C$ onto the upper-level's decision space:

$$
C(X)=\{y:(y, v) \in C\} .
$$

- The constraint region of the lower-level Stampacchia type variational inequality for some given $x \in C(X)$ :

$$
C(x)=\{z: z \in T(x)\} .
$$

- The rational reaction set of the lower-level Stampacchia type variational inequality for some given $x \in C(X)$ :

$$
M(x)=\{z \in C(x): \psi(z, z-v) \leqslant 0, \forall v \in C(x)\} .
$$

- The inducible region of (BVI):

$$
I R=\{(x, z) \in C: z \in M(x), x \in C(X)\} .
$$

It is easy to see that $C(X)=K$, for each fixed $x \in C(X), C(x)=T(x), M(x)=S(x)$ and, the inducible region $I R$ is connected if $K$ and $M(x)$ are connected. Moreover, $I R=\{(x, z): z \in S(x), x \in K\}=\operatorname{Gr}(S)$, the graph of $S$. We say that the induced region $I R$ is the feasible set of the (BVI). It is usually nonconvex and may be disconnected or even empty in the presence of upper-level constraints if the upper-level constraints depends on the lower-level optimal solution (see, Examples 2.1 and 2.2).

Example 2.1 Let $\mathbb{R}=(-\infty,+\infty), K=\left[-1,-\frac{1}{2}\right]$, and $T(x)=[-1, x]$ for $x \in K$. Let $\psi(z, z-v)=\left\langle-(2 z+1)(3 z+2)(4 z+3) \mathrm{e}^{z}, z-v\right\rangle$ and $\Phi(z, x, x-y)=$ $\left\langle-(z+1)(2 z+1) x \mathrm{e}^{z}, x-y\right\rangle$ for $z, v, x, y \in \mathbb{R}$. Simple computation allows that the rational reaction set

$$
M(x)= \begin{cases}\{-1\}, & \text { if } x \in\left[-1,-\frac{3}{4}\right), \\ \left\{-\frac{3}{4}\right\}, & \text { if } x \in\left[-\frac{3}{4},-\frac{2}{3}\right), \\ \left\{-\frac{2}{3},-\frac{3}{4}\right\}, & \text { if } x \in\left[-\frac{2}{3},-\frac{1}{2}\right), \\ \left\{-\frac{1}{2},-\frac{2}{3},-\frac{3}{4}\right\}, & \text { if } x=-\frac{1}{2},\end{cases}
$$

and so, $\left\{(-1, t),\left(-\frac{1}{2},-\frac{1}{2}\right),\left(-\frac{1}{2},-\frac{2}{3}\right),\left(-\frac{1}{2},-\frac{3}{4}\right): t \in\left[-1,-\frac{3}{4}\right)\right\}$ is the solution set of (BVI) (2.1)-(2.2). Then the inducible region $I R$ is disconnected and nonconvex.

Example 2.2 Let $\mathbb{R}^{n}=\mathbb{R}^{m}=\mathbb{R}=(-\infty,+\infty), K=\left(-1,-\frac{1}{2}\right], \quad T(x)=\left[-\frac{3}{2}\right.$, $\left.-1+\frac{x}{2}\right], \Phi(z, x, x-y)=\left\langle-3 x^{2} z-z(x-y), x-y\right\rangle$ and $\psi(z, z-v)=\langle z-v, z-v\rangle$ for all $x, y, z, v \in \mathbb{R}$. After computation, for all $x \in K, M(x)=\emptyset$. Hence, the inducible region $I R$ is empty. 
It is worth noting that the lower-level Stampacchia type variational inequalities (LSVI) contains some existing variational inequalities. For instance, if for each $x \in K, T(x) \equiv \Omega, \psi(z, d)=\langle A z, d\rangle$ for all $z, d \in \mathbb{R}^{m}$, where $A: \mathbb{R}^{m} \rightarrow \mathbb{R}^{m}$ is a vector-valued mapping, then (LSVI) is reduced to the following classical Stampacchia type variational inequality [51]: find $z^{*} \in \Omega$ such that

$$
\left\langle A z^{*}, z^{*}-z\right\rangle \leqslant 0, \quad \forall z \in \Omega .
$$

If for each $x \in K, T(x) \equiv \Omega, \psi(z, d)=g_{-}^{\prime}(z, d)$ for all $z, d \in \mathbb{R}^{m}$, where

$$
g_{-}^{\prime}(z, d)=\liminf _{r \searrow 0} \frac{g(z+r d)-g(z)}{r}
$$

is the lower Dini directional derivative of the function $g: \mathbb{R}^{m} \rightarrow \mathbb{R} \cup\{+\infty\}$ at $z$ in the direction $d$, then (LSVI) is reduced to the following differential type variational inequality studied by Crespi, Ginchev and Rocca $[16,17]$ : find $z^{*} \in \Omega$ such that

$$
g_{-}^{\prime}\left(z^{*}, z^{*}-z\right) \leqslant 0, \quad \forall z \in \Omega .
$$

This shows that the variational inequalities of differential type studied by Crespi, Ginchev and Rocca $[16,17]$ is a special case of the bifunction variational inequality, which are closely related to nonsmooth optimization problems.

We observe that the following relationships among (BVI) and other types of bilevel problems.

(I) If $G: K \rightarrow 2^{\mathbb{R}^{m}}, K$ is a nonempty subset of $\mathbb{R}^{m}$, and $T(x)=K \cap G(x)$ for all $x \in K$, then (BVI) reduces to the following bilevel variational inequality: find $x \in K$ such that

$$
\Phi(z, x, x-y) \leqslant 0, \quad \forall y \in K, z \in S(x),
$$

where $S(x)$ is the solution set of the following variational inequalities defined by bifunction with respect to a parameter $x$ : find $z \in K$ such that $z \in G(x)$ and

$$
\psi(z, z-v) \leqslant 0, \quad \forall v \in G(x) \cap K .
$$

The bilevel model is new, and shows that the lower-level decision maker is not only affected by the action of the upper-level one, but also has its own constraints.

(II) If $\mathbb{R}^{m}=\mathbb{R}^{n}$ and $T(x) \equiv K$ for all $x \in K$, then (BVI) reduces to the following quasi-bilevel variational inequality (shortly (QBVI)) defined by bifunctions: find $x \in S_{\psi}$ such that

$$
\Phi(x, x-y) \leqslant 0, \quad \forall y \in S_{\psi},
$$

where $S_{\psi}$ is the solution set of the following variational inequalities defined by bifunction: find $z \in K$ such that

$$
\psi(z, z-v) \leqslant 0, \quad \forall v \in K
$$

If we set $f(x, y)=\Phi(x, x-y)$ and $h(z, v)=\psi(z, z-v)$ for all $x, y, z$, $v \in \mathbb{R}$, then (QBVI) reduces to a bilevel equilibrium problem of Moudafi [46]. 
The (QBVI) reflects that the lower-level decision maker (follower) firstly makes its decision and feedback all possible decisions to the upper-level decision maker (leader). Thereafter, the leader makes a decision on the possible decisions set of its follower. The leader does not affect the decisions of follower. So, we also call the (QBVI) as two-stage variational inequalities.

(III) If for each $x, y \in K, z, v \in \mathbb{R}^{m}, \Phi(z, x, x-y)=\langle H(z, x), x-y\rangle$ and $\psi(z, z-v)=\langle p(z, v), z-v\rangle$, where $H: \mathbb{R}^{m} \times \mathbb{R}^{n} \rightarrow \mathbb{R}^{n}$ and $H: \mathbb{R}^{m} \times \mathbb{R}^{m} \rightarrow$ $\mathbb{R}^{m}$, then (BVI) reduces to the following bilevel variational inequality: find $x \in K$ such that

$$
\langle H(z, x), x-y\rangle \leqslant 0, \quad \forall y \in K, z \in S(x),
$$

where $S(x)$ is the solution set of the following variational inequalities parameterized in $x$ : find $z \in T(x)$ such that

$$
\langle p(z, v), z-v\rangle \leqslant 0, \quad \forall v \in T(x) .
$$

(IV) If for each $x, y \in K, z \in \mathbb{R}^{m}, \Phi(z, x, x-y)=\phi(z, x)-\phi(z, y)$, then (BVI) reduces to the following optimization problem with variational inequality constraints:

$$
\min _{x, z} \phi(z, x) \quad \text { subject to } x \in K, z \in S_{\psi}(x),
$$

where $S_{\psi}(x)$ is the solution set of the following variational inequalities defined by bifunction with respect to the parameter $x$ : find $z \in T(x)$ such that

$$
\psi(z, z-v) \leqslant 0, \quad \forall v \in T(x) .
$$

(V) If for each $x, y \in K, z, v \in \mathbb{R}^{m}, \Phi(z, x, x-y)=E(z, x, x)-E(z, x, y)$ and $\psi(z, z-v)=Q(z, z)-Q(z, v)$, where for each $z \in \mathbb{R}^{m}, E(z, y, y)=0$ and $Q(v, v)=0$ for all $y \in \mathbb{R}^{n}, v \in \mathbb{R}^{m}$, i.e., $E(z, \cdot, \cdot)$ and $Q(\cdot, \cdot)$ are two equilibrium functions, then (BVI) reduces to the following bilevel equilibrium problem with hierarchical nesting structure:

find $\quad x \in K \quad$ such that $E(z, x, y) \geqslant 0, \quad \forall y \in K, z \in S_{Q}(x)$,

where $S_{Q}(x)$ is the solution set of the following equilibrium problem parameterized in $x$ :

$$
\text { find } \quad z \in T(x) \text { such that } Q(z, v) \geqslant 0 \quad \forall v \in T(x) \text {. }
$$

Obviously, the above bilevel equilibrium problem is distinct from that of Chadli, Mahdioui and Yao [9], Ding [22, 23], Ding, Liou and Yao [26], Dinh and Muu [27] and Moudafi [46].

(VI) If for each $x, y \in K, z, v \in \mathbb{R}^{m}, \Phi(z, x, x-y)=\phi(z, x)-\phi(z, y)$ and $\psi(z, z-v)=\varphi(z)-\varphi(v)$, then (BVI) reduces to the following bilevel programming problem:

$$
\min _{x, z} \phi(z, x) \quad \text { subject to } x \in K, z \in S_{\psi}(x),
$$


where $S_{\psi}(x)$ is the solution set of the following optimization problem parameterized in $x$ :

$$
\min _{z} \varphi(z) \quad \text { subject to } z \in T(x) .
$$

These suffice that the bilevel model of (BVI) is very general bilevel problem with hierarchical structure and includes some existing bilevel problems such as bilevel equilibrium, mathematical programming with variational inequalities and equilibrium constraints and related bilevel programming problems as special cases.

We first recall some definitions and lemmas which are needed in our main results.

Definition 2.1 [30] Let $\Xi$ be a nonempty subset of $\mathbb{R}^{m}$. A bifunction $\psi: \Xi \times \mathbb{R}^{m} \rightarrow$ $\mathbb{R}$ is said to be

(i) monotone if

$$
\psi(x, y-x)+\psi(y, x-y) \leqslant 0, \quad \forall(x, y) \in \Xi \times \Xi
$$

(ii) strictly monotone if

$$
\psi(x, y-x)+\psi(y, x-y)<0, \quad \forall(x, y) \in \Xi \times \Xi, x \neq y
$$

(iii) $\iota$-strongly monotone if there exists a constant $\iota>0$ such that

$$
\psi(x, y-x)+\psi(y, x-y) \leqslant-\iota\|x-y\|^{2}, \quad \forall(x, y) \in \Xi \times \Xi ;
$$

(iv) pseudomonotone if for any $(x, y) \in \Xi \times \Xi$,

$$
\psi(x, y-x) \geqslant 0 \Rightarrow \psi(y, x-y) \leqslant 0 .
$$

It is easy to see that

$$
\begin{aligned}
\iota \text {-strong monotonicity } & \Rightarrow \text { strict monotonicity } \\
& \Rightarrow \text { monotonicity } \Rightarrow \text { pseudomonotonicity. }
\end{aligned}
$$

Definition 2.2 $[30,35]$ Let $\Xi$ be a nonempty subset of $\mathbb{R}^{m}$. A bifunction $\psi: \Xi \times$ $\mathbb{R}^{m} \rightarrow \mathbb{R}$ is said to be subodd if

$$
\psi(x, d)+\psi(x,-d) \geqslant 0, \quad \forall x \in \Xi, d \in \mathbb{R}^{m} .
$$

In the sequel, we give some properties on the suboddness, monotonicity and pseudomonotonicity of $\psi$.

Proposition 2.1 Let $\Xi$ be a nonempty subset of $\mathbb{R}^{m}, \psi: \Xi \times \mathbb{R}^{m} \rightarrow \mathbb{R}$ be subodd and monotone. Then

$$
\psi(x, x-y)+\psi(y, y-x) \geqslant 0, \quad \forall x, y \in \Xi .
$$


Proof For any $x, y \in \Xi$, by the monotonicity of $\psi$, we have

$$
\psi(x, y-x)+\psi(y, x-y) \leqslant 0 .
$$

Since $\psi: \Xi \times \mathbb{R}^{m} \rightarrow \mathbb{R}$ is subodd, we have

$$
\psi(x, y-x)+\psi(x, x-y) \geqslant 0
$$

and

$$
\psi(y, y-x)+\psi(y, x-y) \geqslant 0 .
$$

By adding the left of inequalities (2.4) and (2.5), we get

$$
(\psi(x, y-x)+\psi(y, x-y))+(\psi(x, x-y)+\psi(y, y-x)) \geqslant 0 .
$$

From (2.3) and (2.6), it follows that

$$
\psi(x, x-y)+\psi(y, y-x) \geqslant 0 .
$$

Proposition 2.2 Let $\Xi$ be a nonempty subset of $\mathbb{R}^{m}, \psi: \mathbb{R}^{m} \times \mathbb{R}^{m} \rightarrow \mathbb{R}$ be subodd and strictly monotone. Then

$$
\psi(x, x-y)+\psi(y, y-x)>0, \quad \forall x, y \in \Xi, x \neq y .
$$

Proof For any $x, y \in \Xi, x \neq y$, by the strict monotonicity of $\psi$, we have

$$
\psi(x, y-x)+\psi(y, x-y)<0 .
$$

It follows from (2.6) that

$$
\psi(x, x-y)+\psi(y, y-x) \geqslant-(\psi(x, y-x)+\psi(y, x-y)) .
$$

By virtue of (2.7) and (2.8), it follows that

$$
\psi(x, x-y)+\psi(y, y-x)>0 .
$$

Proposition 2.3 Let $\Xi$ be a nonempty subset of $\mathbb{R}^{m}, \psi: \mathbb{R}^{m} \times \mathbb{R}^{m} \rightarrow \mathbb{R}$ be subodd and $\iota$-strongly monotone. Then

$$
\psi(x, x-y)+\psi(y, y-x) \geqslant \iota\|x-y\|^{2}, \quad \forall x, y \in \Xi .
$$

Proof For any $x, y \in \Xi$, by the $\iota$-strong monotonicity of $\psi$, we have

$$
\psi(x, y-x)+\psi(y, x-y) \leqslant-\imath\|x-y\|^{2} .
$$

Therefore, from (2.8) and (2.9),

$$
\psi(x, x-y)+\psi(y, y-x) \geqslant \iota\|x-y\|^{2} .
$$

Proposition 2.4 Let $\Xi$ be a nonempty subset of $\mathbb{R}^{m}, \psi: \Xi \times \mathbb{R}^{m} \rightarrow \mathbb{R}$ be subodd and pseudomonotone. If $\psi(y, y-x) \leqslant 0$ for $x, y \in \Xi$, then

$$
\psi(x, x-y)+\psi(y, x-y) \geqslant 0 .
$$


Proof Since $\psi(y, y-x) \leqslant 0$ for $x, y \in \Xi$, and from the suboddness of $\psi$, it follows that

$$
\psi(y, x-y) \geqslant 0 .
$$

Note that $\psi: \mathbb{R}^{m} \times \mathbb{R}^{m} \rightarrow \mathbb{R}$ is pseudomonotone. We obtain

$$
\psi(x, y-x) \leqslant 0 .
$$

Again from the suboddness of $\psi$, we have

$$
\psi(x, x-y) \geqslant 0 .
$$

Therefore,

$$
\psi(x, x-y)+\psi(y, x-y) \geqslant 0 .
$$

Definition 2.3 [3] A bifunction $\psi: \mathbb{R}^{m} \times \mathbb{R}^{m} \rightarrow \mathbb{R}$ is said to be

(i) hemicontinuous if for any $z, w \in \mathbb{R}^{m}$ and $r \in[0,1]$, the function $r \mapsto \Psi(r w+$ $(1-r) z, w-z)$ is continuous at $0^{+}$;

(ii) lower-hemicontinuous if for any $z, w, v \in \mathbb{R}^{m}$ and $r \in[0,1]$, the function $g(r)=$ $\Psi(r w+(1-r) z, r w+(1-r) z+v)$ is lower-semicontinuous at $0^{+}$.

Definition 2.4 $[3,6]$ Let $P$ be a Hausdorff topological vector space, and $E$ be a locally convex Hausdorff topological vector space. A set-valued mapping $G: P \rightarrow 2^{E}$ is said to be

(i) upper semicontinuous (shortly, usc) at $v_{0} \in P$ iff, for each open set $V$ with $G\left(v_{0}\right) \subset V$, there exists $\delta>0$ such that

$$
G(v) \subset V, \quad \forall v \in B\left(v_{0}, \delta\right)
$$

(ii) lower semicontinuous (shortly, lsc) at $v_{0} \in P$ iff, for each open set $V$ with $G\left(v_{0}\right) \cap V \neq \emptyset$, there exists $\delta>0$ such that

$$
G(v) \cap V \neq \varnothing, \quad \forall v \in B\left(v_{0}, \delta\right) ;
$$

(iii) closed iff, the graph of $G$ is closed, i.e., the set $\operatorname{Gr}(G)=\{(\zeta, v) \in P \times E: \zeta \in$ $G(v)\}$ is closed in $P \times E$.

We say $G$ is lsc (resp. usc) on $P$ iff it is lsc (resp. usc) at each $v \in P$. $G$ is called continuous on $P$ iff it is both lsc and usc on $P$.

\section{Remark 2.1 [3, 6]}

(i) $G$ is lsc at $v_{0} \in P$ if and only if, for any net $\left\{v_{\alpha}\right\} \subseteq P$ with $v_{\alpha} \rightarrow v_{0}$ and $\zeta_{0} \in$ $G\left(v_{0}\right)$, there exists a net $\left\{\zeta_{\alpha}\right\} \subseteq E$ with $\zeta_{\alpha} \in G\left(v_{\alpha}\right)$ for all $\alpha$, such that $\zeta_{\alpha} \rightarrow \zeta_{0}$;

(ii) If $G$ is compact-valued, then $G$ is usc at $v_{0} \in P$ if and only if, for any net $\left\{v_{\alpha}\right\} \subseteq P$ with $v_{\alpha} \rightarrow v_{0}$ and for any net $\left\{\zeta_{\alpha}\right\} \subseteq E$ with $\zeta_{\alpha} \in G\left(v_{\alpha}\right)$ for all $\alpha$, there exists $\zeta_{0} \in G\left(v_{0}\right)$ and a subnet $\left\{\zeta_{\beta}\right\}$ of $\left\{\zeta_{\alpha}\right\}$ such that $\zeta_{\beta} \rightarrow \zeta_{0}$;

(iii) If $G$ is usc and closed-valued, then $G$ is closed; Conversely, if $G$ is closed and $E$ is compact, then $G$ is usc. 
Definition 2.5 $[3,4]$ Let $K$ be a nonempty convex subset of $\mathbb{R}^{n}$. A set-valued mapping $T: K \rightarrow 2^{\mathbb{R}^{m}}$ is said to be

(i) convex if,

$$
\lambda T\left(x_{1}\right)+(1-\lambda) T\left(x_{2}\right) \subseteq T\left(\lambda x_{1}+(1-\lambda) x_{2}\right), \quad \forall x_{1}, x_{2} \in K, \lambda \in[0,1] ;
$$

(ii) convex-valued (compact-valued, closed-valued) if, the images $T(x)$ of all points $x \in K$ are convex (compact, closed).

Remark 2.2 It is well-known that $T: K \rightarrow 2^{\mathbb{R}^{m}}$ is convex if and only if the graph $\operatorname{Gr}(T)$ is convex subset of $\mathbb{R}^{n} \times \mathbb{R}^{m}$, where $\operatorname{Gr}(T)=\{(y, z): z \in T(y)\}$ is the graph of $T$; If $T$ is a convex mapping, then $T$ is convex-valued. Indeed, for each $y \in K$, any $z_{1}, z_{2} \in T(y)$, we have $\left(y, z_{1}\right),\left(y, z_{2}\right) \in \operatorname{Gr}(T)$ and so, $\lambda z_{1}+(1-\lambda) z_{2} \in T(\lambda y+$ $(1-\lambda) y)=T(y)$ for all $\lambda \in[0,1]$.

Proposition 2.5 Let $K$ be a nonempty closed and convex subset of $\mathbb{R}^{n}$ and $T: K \rightarrow$ $2^{\mathbb{R}^{m}}$ be closed and convex. Then $C$ is closed and convex.

Proof Let $\left\{\left(y_{k}, v_{k}\right)\right\} \subseteq C$ such that $\left(y_{k}, v_{k}\right) \rightarrow\left(y_{0}, v_{0}\right)$. Then $v_{k} \in T\left(y_{k}\right)$. By the closedness of $T, v_{0} \in T\left(y_{0}\right)$. Thus $\left(y_{0}, v_{0}\right) \in C$, i.e., $C$ is closed.

On the other hand, let $\left(y_{1}, v_{1}\right),\left(y_{2}, v_{2}\right) \in C$. Then $v_{1} \in T\left(y_{1}\right)$ and $v_{2} \in T\left(y_{2}\right)$. By the convexity of $T$ and $K, \lambda y_{1}+(1-\lambda) y_{2} \in K$ and $\lambda v_{1}+(1-\lambda) v_{2} \in T\left(\lambda y_{1}+\right.$ $\left.(1-\lambda) y_{2}\right)$ for all $\lambda \in[0,1]$. Consequently, $\left(\lambda y_{1}+(1-\lambda) y_{2}, \lambda v_{1}+(1-\lambda) v_{2}\right) \in C$. Therefore, $C$ is closed and convex.

Definition 2.6 [29] Let $D$ be a nonempty subset of a linear space $X$. A set-valued mapping $H: D \rightarrow 2^{X}$ is said to be a KKM mapping if for any finite subset $\left\{z_{1}, z_{2}, \cdots, z_{k}\right\}$ of $D$,

$$
\operatorname{co}\left\{z_{1}, z_{2}, \cdots, z_{k}\right\} \subseteq \bigcup_{j=1}^{k} H\left(z_{j}\right),
$$

where $\operatorname{co}\left\{z_{1}, z_{2}, \cdots, z_{k}\right\}$ denotes the convex hull of $\left\{z_{1}, z_{2}, \cdots, z_{k}\right\}$.

Lemma 2.1 [29] Let $D$ be a nonempty convex subset of a Hausdorff topological vector space $X$ and let $H: D \rightarrow 2^{X}$ be a KKM mapping with closed values. If there exists some $z_{0} \in D$ such that $H\left(z_{0}\right)$ is a compact subset of $X$, then there exists $z^{*} \in D$ such that $z^{*} \in H(z)$ for all $z \in K$, i.e., $\bigcap_{z \in D} H(z) \neq \emptyset$.

\section{Existence Results for (BVI)}

In this section, we investigate the sufficient optimality conditions for (LSVI) and (BVI) when the rational reaction set $M(x)$ is a singleton under some suitable conditions, and discuss some topological properties of their solutions.

Theorem 3.1 Let $T: K \rightarrow 2^{\mathbb{R}^{m}}$ be closed and convex-valued, $\psi: \mathbb{R}^{m} \times \mathbb{R}^{m} \rightarrow \mathbb{R}$ be subodd and pseudomonotone. Assume that the following conditions hold: 
(i) the function $z \mapsto \psi(\cdot, \cdot-z)$ is concave, i.e., $z \mapsto-\psi(\cdot, \cdot-z)$ is convex;

(ii) for each $z \in \mathbb{R}^{m}, \psi(\cdot, \cdot-z)$ is lower-hemicontinuous.

Then for each $x \in K$, the following problems are equivalent:

(a) find $z^{*} \in T(x)$ such that

$$
\psi\left(z^{*}, z^{*}-z\right) \leqslant 0, \quad \forall z \in T(x) ;
$$

(b) find $z^{*} \in T(x)$ such that

$$
\psi\left(z, z^{*}-z\right) \leqslant 0, \quad \forall z \in T(x) .
$$

Proof (a) $\Rightarrow(\mathrm{b})$ : Suppose that $z^{*} \in T(x)$ is a solution of (a), i.e., $z^{*} \in M(x)$. We have

$$
\psi\left(z^{*}, z^{*}-z\right) \leqslant 0, \quad \forall z \in T(x) .
$$

Since $\psi: \mathbb{R}^{m} \times \mathbb{R}^{m} \rightarrow \mathbb{R}$ is subodd,

$$
\psi\left(z^{*}, z^{*}-z\right)+\psi\left(z^{*}, z-z^{*}\right) \geqslant 0
$$

and so, $\psi\left(z^{*}, z-z^{*}\right) \geqslant 0$. By the pseudomonotonicity of $\psi$, we obtain

$$
\psi\left(z, z^{*}-z\right) \leqslant 0 .
$$

This yields that $z^{*}$ is a solution of (b).

(b) $\Rightarrow$ (a): Suppose that $z^{*} \in T(x)$ is a solution of (b). Then

$$
\psi\left(z, z^{*}-z\right) \leqslant 0, \quad \forall z \in T(x) .
$$

By the suboddness of $\psi$,

$$
\psi\left(z, z^{*}-z\right)+\psi\left(z, z-z^{*}\right) \geqslant 0, \quad \forall z \in T(x) .
$$

Moreover, we get

$$
\psi\left(z, z-z^{*}\right) \geqslant 0, \quad \forall z \in T(x) .
$$

Let $z_{t}=t z+(1-t) z^{*}$ for all $t \in(0,1)$. Then $z_{t} \in T(x)$ and, from $\psi\left(z_{t}, 0\right)=0$, we have

$$
0=\psi\left(z_{t}, z_{t}-z_{t}\right) \geqslant t \psi\left(z_{t}, z_{t}-z\right)+(1-t) \psi\left(z_{t}, z_{t}-z^{*}\right) .
$$

Furthermore, we have

$$
\psi\left(z_{t}, z_{t}-z\right) \leqslant 0, \quad \forall z \in T(x) .
$$

Let $g(t)=\psi\left(z_{t}, z_{t}-z\right)$. By condition (ii), $g$ is lower-semicontinuous and so,

$$
\psi\left(z^{*}, z^{*}-z\right)=g(0) \leqslant \liminf _{t \searrow 0} g(t)=\liminf _{t \searrow 0} \psi\left(z_{t}, z_{t}-z\right) \leqslant 0, \quad \forall z \in T(x) .
$$

Therefore, $z^{*} \in M(x)$.

Remark 3.1 Compared with Lemma 5.1 of Fang and Hu [30], we removed the positive homogeneity of $\psi$ with respect to the second variable. 
Theorem 3.2 Let $T: K \rightarrow 2^{\mathbb{R}^{m}}$ be closed convex-valued, $\psi: \mathbb{R}^{m} \times \mathbb{R}^{m} \rightarrow \mathbb{R}$ be subodd and monotone. Assume that the following conditions hold:

(i) the function $z \mapsto \psi(\cdot, \cdot-z)$ is concave and usc;

(ii) for each $z \in \mathbb{R}^{m}, \psi(\cdot, \cdot-z)$ is lower-hemicontinuous.

Then for each $x \in K$, the solution set $M(x)$ of (LSVI) is closed and convex.

Proof Let $x \in K$. Clearly, $M(x) \subseteq T(x)$. Since $T: K \rightarrow 2^{\mathbb{R}^{m}}$ is closed convexvalued, we obtain that $T(x)$ is a closed and convex subset of $\mathbb{R}^{m}$. Without loss of generality, assume that $M(x)$ is nonempty. We divide the rest proof into two steps.

Step 1 . We show that $M(x)$ is convex. Take any $z^{\prime}, z^{\prime \prime} \in M(x)$, we have

$$
\psi\left(z^{\prime}, z^{\prime}-z\right) \leqslant 0, \quad \forall z \in T(x)
$$

and

$$
\psi\left(z^{\prime \prime}, z^{\prime \prime}-z\right) \leqslant 0, \quad \forall z \in T(x) .
$$

Set $z_{\lambda}=\lambda z^{\prime}+(1-\lambda) z^{\prime \prime}$ for all $\lambda \in[0,1]$. Then $z_{\lambda} \in T(x)$. Since $\psi: \mathbb{R}^{m} \times \mathbb{R}^{m} \rightarrow \mathbb{R}$ is subodd and monotone, by Proposition 2.1, we get

$$
\psi\left(z^{\prime}, z^{\prime}-z\right)+\psi\left(z, z-z^{\prime}\right) \geqslant 0, \quad \forall z \in T(x)
$$

and

$$
\psi\left(z^{\prime \prime}, z^{\prime \prime}-z\right)+\psi\left(z, z-z^{\prime \prime}\right) \geqslant 0, \quad \forall z \in T(x) .
$$

From (3.1)-(3.4), it follows that

$$
\psi\left(z, z-z^{\prime}\right) \geqslant 0, \quad \forall z \in T(x)
$$

and

$$
\psi\left(z, z-z^{\prime \prime}\right) \geqslant 0, \quad \forall z \in T(x) .
$$

In view of condition (i), we have

$$
\psi\left(z, z-z_{\lambda}\right) \geqslant \lambda \psi\left(z, z-z^{\prime}\right)+(1-\lambda) \psi\left(z, z-z^{\prime \prime}\right) \geqslant 0, \quad \forall z \in T(x) .
$$

Put $z_{t}=t z+(1-t) z_{\lambda}$ for all $t \in(0,1)$ and $z \in T(x)$. So, $z_{t} \in T(x)$. Then

$$
0=\psi\left(z_{t}, 0\right)=\psi\left(z_{t}, z_{t}-z_{t}\right) \geqslant t \psi\left(z_{t}, z_{t}-z\right)+(1-t) \psi\left(z_{t}, z_{t}-z_{\lambda}\right) .
$$

From (3.5) and (3.6), we have

$$
t \psi\left(z_{t}, z_{t}-z\right) \leqslant 0,
$$

that is, $\psi\left(z_{t}, z_{t}-z\right) \leqslant 0$ for all $z \in T(x)$. This, together with condition (ii), yields that

$$
\psi\left(z_{\lambda}, z_{\lambda}-z\right) \leqslant \liminf _{t \searrow 0} \psi\left(z_{t}, z_{t}-z\right) \leqslant 0, \quad \forall z \in T(x),
$$

that is, $\psi\left(z_{\lambda}, z_{\lambda}-z\right) \leqslant 0$ and so, $z_{\lambda} \in M(x)$. Thus $M(x)$ is convex. 
Step 2. We show that $M(x)$ is closed. Take any sequence $\left\{z_{k}\right\} \subseteq M(x)$ with $z_{k} \rightarrow z_{0}$. Then $z_{0} \in T(x)$ and

$$
\psi\left(z_{k}, z_{k}-z\right) \leqslant 0, \quad \forall z \in T(x) .
$$

Since $\psi: \mathbb{R}^{m} \times \mathbb{R}^{m} \rightarrow \mathbb{R}$ is subodd and monotone, from Proposition 2.1, we have

$$
\psi\left(z_{k}, z_{k}-z\right)+\psi\left(z, z-z_{k}\right) \geqslant 0, \quad \forall z \in T(x)
$$

and so,

$$
\psi\left(z, z-z_{k}\right) \geqslant 0, \quad \forall z \in T(x) .
$$

This, together with condition (i), shows that

$$
\psi\left(z, z-z_{0}\right) \geqslant \limsup _{k \rightarrow \infty} \psi\left(z, z-z_{k}\right) \geqslant 0, \quad \forall z \in T(x) .
$$

Similarly to Step 1 , set $z_{l}=l z+(1-l) z_{0}$ for all $l \in(0,1)$. Clearly, $z_{l} \in T(x)$. Consequently,

$$
0=\psi\left(z_{l}, 0\right)=\psi\left(z_{l}, z_{l}-z_{l}\right) \geqslant l \psi\left(z_{l}, z_{l}-z\right)+(1-l) \psi\left(z_{l}, z_{l}-z_{0}\right) .
$$

This implies that

$$
\psi\left(z_{l}, z_{l}-z\right) \leqslant 0, \quad \forall z \in T(x) .
$$

Take into account the condition (ii), we derive that

$$
\psi\left(z_{0}, z_{0}-z\right) \leqslant \liminf _{t \searrow 0} \psi\left(z_{l}, z_{l}-z\right) \leqslant 0, \quad \forall z \in T(x) .
$$

This shows that $z_{0} \in M(x)$. Therefore, $M(x)$ is closed.

Next, we prove the existence of solution to (LSVI).

Theorem 3.3 Let $T: K \rightarrow 2^{\mathbb{R}^{m}}$ be convex and compact-valued, $\psi: \mathbb{R}^{m} \times \mathbb{R}^{m} \rightarrow \mathbb{R}$ be subodd and pseudomonotone. Assume that the following conditions hold:

(i) the function $z \mapsto \psi(\cdot, \cdot-z)$ is concave and usc;

(ii) for each $z \in \mathbb{R}^{m}, \psi(\cdot, \cdot-z)$ is lower-hemicontinuous;

(iii) for each $w, z \in \mathbb{R}^{m}, \psi(w, \cdot-z)$ is lsc.

Then for each $x \in K$, the solution set $M(x)$ of (LSVI) is nonempty and compact.

Proof Since $T: K \rightarrow 2^{\mathbb{R}^{m}}$ is convex and compact-valued, for each $x \in K, T(x)$ is a convex and compact subset of $\mathbb{R}^{m}$, define two set-valued mappings $\Gamma_{x}, \Upsilon_{x}: T(x) \rightarrow$ $2^{\mathbb{R}^{m}}$ by, for each $z \in T(x)$,

$$
\Gamma_{x}(z)=\{v \in T(x): \psi(v, v-z) \leqslant 0\}
$$

and

$$
\Upsilon_{x}(z)=\{v \in T(x): \psi(z, v-z) \leqslant 0\}
$$


By Theorem 3.1, we have

$$
M(x)=\bigcap_{z \in T(x)} \Gamma_{x}(z)=\bigcap_{z \in T(x)} \Upsilon_{x}(z)
$$

and

$$
\Gamma_{x}(z) \subseteq \Upsilon_{x}(z)
$$

Next, we divide the rest proof into two steps:

Step 1. We show that $\Gamma_{x}$ is a KKM mapping on $T(x)$. Suppose by contradiction that $\Gamma_{x}$ is not a KKM mapping on $T(x)$. Then there exist a finite subset $\left\{v_{1}, v_{2}, \cdots, v_{k}\right\}$ of $T(x)$ such that

$$
\operatorname{co}\left\{v_{1}, v_{2}, \cdots, v_{k}\right\} \nsubseteq \bigcup_{j=1}^{k} \Gamma_{x}\left(v_{j}\right)
$$

That is, there exists some $v^{\prime} \in \operatorname{co}\left\{v_{1}, v_{2}, \cdots, v_{k}\right\}$, i.e., $v^{\prime}=\sum_{j=1}^{k} \lambda_{j} v_{j}$ for some $\lambda_{j} \in[0,1], j=1,2, \cdots, k$ and $\sum_{j=1}^{k} \lambda_{j}=1$ such that $\nu^{\prime} \notin \Gamma_{x}\left(v_{j}\right), j=1,2, \cdots, k$. Moreover, we have

$$
\psi\left(v^{\prime}, v^{\prime}-v_{j}\right)>0, \quad j=1,2, \cdots, k
$$

Therefore,

$$
\lambda_{j} \psi\left(v^{\prime}, v^{\prime}-v_{j}\right) \geqslant 0, \quad j=1,2, \cdots, k,
$$

and, for some $i \in\{1,2, \cdots, k\}$,

$$
\lambda_{i} \psi\left(v^{\prime}, v^{\prime}-v_{i}\right)>0 .
$$

In view of condition (i), from (3.8) and (3.9), we have

$0=\psi\left(v^{\prime}, 0\right)=\psi\left(v^{\prime}, v^{\prime}-v^{\prime}\right)=\psi\left(v^{\prime}, v^{\prime}-\sum_{j=1}^{k} \lambda_{j} v_{j}\right) \geqslant \sum_{j=1}^{k} \lambda_{j} \psi\left(v^{\prime}, v^{\prime}-v_{j}\right)>0$,

which is a contradiction. Therefore, $\Gamma_{x}$ is a KKM mapping on $T(x)$. It follows from (3.7) that $\Upsilon_{x}$ is also a KKM mapping on $T(x)$.

Step 2. We show that $\Upsilon_{x}$ is closed-valued. For each $z \in T(x)$, let any sequence $\left\{v_{n}\right\} \subseteq \Upsilon_{x}(z)$ such that $v_{n} \rightarrow v_{0}$. Then

$$
\psi\left(z, v_{n}-z\right) \leqslant 0 .
$$

By condition (iii), we have

$$
\psi\left(z, v_{0}-z\right) \leqslant \liminf _{n \rightarrow+\infty} \psi\left(z, v_{n}-z\right) \leqslant 0 .
$$

This shows that $\nu_{0} \in \Upsilon_{x}(z)$ and so, $\Upsilon_{x}(z)$ is closed. Observe that $T(x)$ is compact and $\Upsilon_{x}(z) \subseteq T(x)$. Therefore, $\Upsilon_{x}(z)$ is compact. By Lemma 2.1, we have

$$
M(x)=\bigcap_{z \in T(x)} \Gamma_{x}(z)=\bigcap_{z \in T(x)} \Upsilon_{x}(z) \neq \emptyset
$$


and so, $M(x)$ is compact. To sum up, for each $x \in K$, the solution set $M(x)$ of (LSVI) is nonempty and compact.

Next, we show that the inducible region $I R$ is nonempty and compact.

Corollary 3.1 Let $K$ be a nonempty compact and convex subset of $\mathbb{R}^{n}, T: K \rightarrow$ $2^{\mathbb{R}^{m}}$ be closed convex and compact-valued, and $\psi: \mathbb{R}^{m} \times \mathbb{R}^{m} \rightarrow \mathbb{R}$ be subodd and pseudomonotone. Assume that for each $z \in \mathbb{R}^{m}, \psi(\cdot, \cdot-z)$ is convex and lsc, and conditions (i), (iii) of Theorem 3.3 hold. Then I $R$ is nonempty compact.

Proof It immediately follows from Proposition 2.5 and Theorem 3.3 that $I R$ is nonempty and compact.

If the conditions of Theorem 3.3 are strengthened, we can get the uniqueness of solution to (LSVI).

Theorem 3.4 Let $T: K \rightarrow 2^{\mathbb{R}^{m}}$ be convex and compact-valued, $\psi: \mathbb{R}^{m} \times \mathbb{R}^{m} \rightarrow \mathbb{R}$ be subodd and strictly monotone. Assume that the following conditions hold:

(i) the function $z \mapsto \psi(\cdot, \cdot-z)$ is concave and usc;

(ii) for each $z \in \mathbb{R}^{m}, \psi(\cdot, \cdot-z)$ is lower-hemicontinuous;

(iii) for each $w, z \in \mathbb{R}^{m}, \psi(w, \cdot-z)$ is lsc.

Then for each $x \in K$, the solution set $M(x)$ of (LSVI) is a singleton.

Proof For each $x \in K$, from Theorem 3.3, the solution set $M(x) \neq \emptyset$. Suppose that the solution set $M(x)$ is not a singleton. That is, there exist $z_{1}^{*}, z_{2}^{*} \in M(x)$ such that $z_{1}^{*} \neq z_{2}^{*}$. Then

$$
\psi\left(z_{1}^{*}, z_{1}^{*}-z\right) \leqslant 0, \quad \forall z \in T(x)
$$

and

$$
\psi\left(z_{2}^{*}, z_{2}^{*}-z\right) \leqslant 0, \quad \forall z \in T(x)
$$

Moreover, we have

$$
\psi\left(z_{1}^{*}, z_{1}^{*}-z_{2}^{*}\right) \leqslant 0
$$

and

$$
\psi\left(z_{2}^{*}, z_{2}^{*}-z_{1}^{*}\right) \leqslant 0 .
$$

According to Proposition 2.2, we have

$$
\psi\left(z_{1}^{*}, z_{1}^{*}-z_{2}^{*}\right)+\psi\left(z_{2}^{*}, z_{2}^{*}-z_{1}^{*}\right)>0 .
$$

It follows from (3.10) and (3.12) that

$$
\psi\left(z_{2}^{*}, z_{2}^{*}-z_{1}^{*}\right)>0,
$$

which contradicts (3.11). 
Remark 3.2 Compared with Lemma 5.2 of Fang and Hu [30], the strong monotonicity is relaxed to the strict monotonicity, and the positive homogeneity of $\psi$ with respect to the second variable is also removed. Moreover, the suboddness and strict monotonicity of $\psi$ could not be relaxed to the monotonicity (see, Example 3.1), the strict monotonicity of $\psi$ could not be relaxed to the monotonicity (see, Example 3.2) in Theorem 3.4 .

Example 3.1 Let $\mathbb{R}^{n}=\mathbb{R}^{m}=\mathbb{R}=(-\infty,+\infty), K=[-1,1]$. For each $x \in K$, $T(x)=[x, 1]$ and $\psi(z, z-v)=\langle-(v+z), z-v\rangle$ for $z, v \in T(x)$. Clearly, $\psi$ is monotone and is not subodd and strictly monotone. Moreover, the conditions (i)-(iii) of Theorem 3.4 are satisfied. However, $M(x)=\{-1,1\}$ for $x=-1$.

Example 3.2 Let $\mathbb{R}^{n}=\mathbb{R}^{m}=\mathbb{R}=(-\infty,+\infty), K=[-1,1]$. For each $x \in K$, $T(x)=[x, 1]$ and $\psi(z, z-v) \equiv 0$ for $z, v \in T(x)$. Clearly, $\psi$ is monotone and subodd. Moreover, the conditions (i)-(iii) of Theorem 3.4 are satisfied. But $M(x)=$ $[x, 1]$ for all $x \in K \backslash\{1\}$.

The following result is a direct consequence of Theorem 3.4.

Corollary 3.2 Let $T: K \rightarrow 2^{\mathbb{R}^{m}}$ be convex and compact-valued, $\psi: \mathbb{R}^{m} \times \mathbb{R}^{m} \rightarrow$ $\mathbb{R}$ be subodd and l-strongly monotone. Assume that the conditions (i), (ii) and (iii) of Theorem 3.4 hold. Then for each $x \in K$, the solution set $M(x)$ of (LSVI) is a singleton.

From Theorems 3.2-3.4, it is easy to obtain the following existence theorem of (BVI).

Theorem 3.5 Let $K$ be a compact and convex subset of $\mathbb{R}^{n}$ and $\Phi: \mathbb{R}^{m} \times \mathbb{R}^{n} \times \mathbb{R}^{n} \rightarrow$ $\mathbb{R}$ be a function. Assume that all conditions of Theorem 3.4 are satisfied, and for each $z \in \mathbb{R}^{m}$, the following conditions hold:

(i) the function $\Phi(z, \cdot, \cdot)$ is subodd and pseudomonotone;

(ii) the function $y \mapsto \Phi(z, \cdot, \cdot-y)$ is concave and usc;

(iii) for each $y \in \mathbb{R}^{n}, \Phi(z, \cdot, \cdot-y)$ is lower-hemicontinuous;

(iv) for each $x, y \in \mathbb{R}^{m}, \Phi(z, x, \cdot-y)$ is lsc.

Then the solution set $\Theta$ of (BVI) is nonempty and compact.

Proof By Theorem 3.4, we know that for each $x \in K$, the rational reaction set $M(x)$ of (LSVI) is a singleton. The rest proof is similar to that of Theorem 3.3 and so omitted here.

Remark 3.3 Generally speaking, the optimal solution of (BVI) is not unique, even though the lower-level variational inequality has a unique solution.

Example 3.3 Let $\mathbb{R}^{n}=\mathbb{R}^{m}=\mathbb{R}=(-\infty,+\infty), K=\left[-1,-\frac{1}{2}\right], T(x)=[x, 1]$ for $x \in K$, and let $\psi(z, z-v)=\left\langle\mathrm{e}^{z}, z-v\right\rangle$ and $\Phi(z, x, x-y)=\left\langle-(z+1)(2 z+1) x \mathrm{e}^{z}\right.$, 
$x-y\rangle$ for $z, v \in T(x), x, y \in K$, where $\mathrm{e}$ is the base of natural logarithms. It is easy to verify that all conditions of Theorem 3.5 are satisfied. Simple computation allows that for each $x \in K$, the rational reaction set $M(x)=\{x\}$ and so, $\Theta=\left\{\left(-\frac{1}{2},-\frac{1}{2}\right),(-1,-1)\right\}$. This shows that if the upper-level decision maker gives a decision $x$, the rational reaction set $M(x)$ of (LSVI) is a singleton and the optimal solution set $\Theta$ of (BVI) is nonempty and compact. But the optimal solution of (BVI) is not unique.

Example 3.4 Let $\mathbb{R}^{n}=\mathbb{R}^{m}=\mathbb{R}=(-\infty,+\infty), K=\left[-1,-\frac{1}{2}\right], T(x)=[x, 1]$ for $x \in K$, and let $\psi(z, z-v)=\left\langle\mathrm{e}^{z}, z-v\right\rangle$ and $\Phi(z, x, x-y)=0$ for $x, y \in K, z \in T(x)$. By computation, for each $x \in K$, the rational reaction set $M(x)=\{x\}$ and $\Theta=$ $\left\{\left(-\frac{1}{2},-\frac{1}{2}\right),(-1,-1)\right\}$. That is, the optimal solution set $\Theta$ of (BVI) is not a singleton.

\section{A Penalty Function Method for (BVI)}

In this section, we shall transform the (BVI) (2.1)-(2.2) into one-level variational inequalities by the gap function of (LSVI). We also show that (BVI) is equivalent to a one-level variational inequality under some suitable conditions.

We now define the functions $g: \mathbb{R}^{n} \times \mathbb{R}^{n} \rightarrow \mathbb{R}$ and $f: \mathbb{R}^{m} \times \mathbb{R}^{n} \rightarrow \mathbb{R}$ by, respectively,

$$
g_{\gamma}(x, x-y)=\inf _{z \in T(x)}[\Phi(z, x, x-y)+\gamma f(z, x)], \quad x, y \in \mathbb{R}^{n}
$$

for some $\gamma>0$, and

$$
f(z, x)=\sup _{v \in T(x)} \psi(z, z-v), \quad(z, x) \in \mathbb{R}^{m} \times \mathbb{R}^{n} .
$$

Remark 4.1 For each $x \in K, z \in M(x)$ if and only if $f(z, x)=0$; Moreover, $f(z, x) \geqslant 0$ for all $z \in T(x)$. We say that the function $f$ is a parametric gap function. So, $f$ will be referred as a penalty function.

For $\gamma>0$, we consider the following parametric variational inequality defined by bifunction $(\mathrm{PVI})_{\gamma}$ : find $x \in K$ such that

$$
g_{\gamma}(x, x-y) \leqslant 0, \quad \forall y \in K .
$$

Denote the solution set of $(\mathrm{PVI})_{\gamma}$ by $\mathcal{S}_{\gamma}$.

Theorem 4.1 $(x, z) \in \Theta \Rightarrow x \in \mathcal{S}_{\gamma}$.

Proof Assume that $(x, z) \in \Theta$. Then $x \in K, z \in T(x)$,

$$
\psi(z, z-v) \leqslant 0, \quad \forall v \in T(x)
$$

and

$$
\Phi(z, x, x-y) \leqslant 0, \quad \forall(y, z) \in I R .
$$


Moreover, we have

$$
f(z, x)=\sup _{\nu \in T(x)} \psi(z, z-v) \leqslant 0, \quad(x, z) \in I R .
$$

Therefore, for any $\gamma>0$,

$$
g_{\gamma}(x, x-y)=\inf _{z \in T(x)}[\Phi(z, x, x-y)+\gamma f(z, x)] \leqslant 0, \quad \forall y \in K .
$$

This implies that $x$ is also a solution of $(\mathrm{PVI})_{\gamma}$ and so, $x \in \mathcal{S}_{\gamma}$.

Theorem 4.2 Assume that all conditions of Theorem 3.4 hold. If $x \in \mathcal{S}_{\gamma}$, then for each $z \in M(x),(x, z) \in \Theta$.

Proof Let $x \in \mathcal{S}_{\gamma}$. Then, there exists some $\gamma>0$ such that

$$
g_{\gamma}(x, x-y)=\inf _{z \in T(x)}[\Phi(z, x, x-y)+\gamma f(z, x)] \leqslant 0, \quad \forall y \in K .
$$

Since $T: K \rightarrow 2^{\mathbb{R}^{m}}$ is compact-valued, there exists $z_{\gamma} \in T(x)$ such that

$$
\Phi\left(z_{\gamma}, x, x-y\right)+\gamma f\left(z_{\gamma}, x\right) \leqslant 0, \quad \forall y \in K .
$$

In view of $\Phi\left(z_{\gamma}, x, x-x\right)=\Phi\left(z_{\gamma}, x, 0\right)=0$, from (4.2), $f\left(z_{\gamma}, x\right) \leqslant 0$. Moreover, we have

$$
f\left(z_{\gamma}, x\right)=\sup _{\nu \in T(x)} \psi\left(z_{\gamma}, z_{\gamma}-v\right) \leqslant 0
$$

that is,

$$
\psi\left(z_{\gamma}, z_{\gamma}-v\right) \leqslant 0, \quad \forall v \in T(x) .
$$

Consequently, $\left(x, z_{\gamma}\right) \in I R$. By Theorem 3.4, $M(x)=\left\{z_{\gamma}\right\}$. Again from Remark 4.1 and (4.2), we have

$$
\Phi\left(z_{\gamma}, x, x-y\right) \leqslant 0, \quad \forall y \in K
$$

and hence,

$$
\Phi\left(z_{\gamma}, x, x-y\right) \leqslant 0, \quad \forall\left(y, z_{\gamma}\right) \in I R .
$$

Therefore $\left(x, z_{\gamma}\right) \in \Theta$.

From Theorems 3.5, 4.1 and 4.2, we can obtain the existence of solution for (PVI).

Corollary 4.1 If all conditions of Theorem 3.4 hold, then $(x, z) \in \Theta \Leftrightarrow x \in \mathcal{S}_{\gamma}$.

Corollary 4.2 If all conditions of Theorem 3.5 are satisfied, then $\mathcal{S}_{\gamma}$ is nonempty and compact. 


\section{Algorithm and Convergence Analysis}

In this section, we first present an algorithm for (BVI) (2.1)-(2.2) from the theoretical point of view, and then consider the limiting behavior of a sequence generated by the algorithm. We shall solve (BVI) (2.1)-(2.2) by solving a sequence of approximation problems $(\mathrm{PVI})_{\gamma}(4.1)$ with $\gamma>0$. Based on this fact, we propose the following iterative algorithm to compute the approximation solution of (BVI) (2.1)-(2.2).

\section{Algorithm 5.1}

Step 1. Take $\left\{\gamma_{k}\right\}_{k \in \mathbb{Z}^{+}} \subset(0,+\infty)$ and $\gamma_{k} \nearrow+\infty$, where $\mathbb{Z}^{+}$is the set of all nonnegative integers. Choose $x_{0} \in K$ arbitrarily. Let the tolerance $\varepsilon \geqslant 0, k=0$ and go to Step 2.

Step 2. Given $x_{k} \in K$, if

$$
\exists z_{k} \in T\left(x_{k}\right), \quad \Phi\left(z_{k}, x_{k}, x_{k}-y\right)+\gamma_{k} f\left(z_{k}, x_{k}\right) \leqslant \varepsilon, \quad \forall y \in K,
$$

then stop and output $x_{k}$ and $z_{k}$. Otherwise, take $z_{k} \in T\left(x_{k}\right)$ such that

$$
f\left(z_{k}, x_{k}\right) \leqslant f\left(z, x_{k}\right), \quad \forall z \in T\left(x_{k}\right),
$$

and go to Step 3.

Step 3. Compute $x_{k+1} \in K$.

Find $z_{k+1} \in T\left(x_{k+1}\right)$ such that

$$
f\left(z_{k+1}, x_{k+1}\right) \leqslant \max \left\{0, \frac{1}{\gamma_{k}} f\left(z_{k}, x_{k}\right)\right\},
$$

and

$$
\Phi\left(z_{k+1}, x_{k+1}, x_{k+1}-y\right) \leqslant \varepsilon, \quad \forall y \in K .
$$

Step 4. Set $k:=k+1$ and go to Step 2.

Let us consider the behavior of a sequence $\left\{\left(x_{k}, z_{k}\right)\right\}$ generated by Algorithm 5.1.

Theorem 5.1 Assume that all conditions of Theorem 3.5 hold, for each $y \in \mathbb{R}^{m}$, the function $\Phi(\cdot, \cdot, \cdot-y)$ is lsc and $T: K \rightarrow 2^{\mathbb{R}^{m}}$ is continuous. Then the sequence $\left\{\left(x_{k}, z_{k}\right)\right\}$ generated by Algorithm 5.1 converges to some solution of $(B V I)$.

Proof By Algorithm 5.1, we have

$$
f\left(z_{k+1}, x_{k+1}\right) \leqslant \max \left\{0, \frac{1}{\gamma_{k}} f\left(z_{k}, x_{k}\right)\right\} .
$$

This shows that

$$
f\left(z_{k+1}, x_{k+1}\right) \leqslant \max \left\{0, \prod_{j=0}^{k} \frac{1}{\gamma_{j}} f\left(z_{0}, x_{0}\right)\right\} .
$$


Observe that $\gamma_{k} \nearrow+\infty$. Set $\hat{\gamma}=1$. Then there exists a nonnegative integer $\bar{k}$ such that $\gamma_{\bar{k}} \geqslant \hat{\gamma}$ and so, $\prod_{i=0}^{\bar{k}} \frac{1}{\gamma_{i}}$ is a finite positive number. For any $k, k>\bar{k}$, from (5.5),

$$
f\left(z_{k+1}, x_{k+1}\right) \leqslant \max \left\{0, \prod_{i=0}^{\bar{k}} \frac{1}{\gamma_{i}} \cdot \prod_{j=\bar{k}+1}^{k} \frac{1}{\gamma_{j}} f\left(z_{0}, x_{0}\right)\right\} .
$$

Since $T: K \rightarrow 2^{\mathbb{R}^{m}}$ is convex compact-valued, from condition (i) of Theorem 3.4, we know that $f\left(z_{0}, x_{0}\right)$ is finite. This, together with $\gamma_{k} \nearrow+\infty$, yields that $\prod_{j=\bar{k}+1}^{k} \frac{1}{\gamma_{j}} f\left(z_{0}, x_{0}\right) \searrow 0$. By (5.6), we have

$$
\limsup _{k \rightarrow+\infty} f\left(z_{k+1}, x_{k+1}\right) \leqslant \limsup _{k \rightarrow+\infty} \max \left\{0, \prod_{j=0}^{k} \frac{1}{\gamma_{j}} f\left(z_{0}, x_{0}\right)\right\}=0 .
$$

Observe that $\left\{x_{k}\right\}_{k \in \mathbb{Z}^{+}} \subseteq K$ is compact. Without loss of generality, let $x_{k} \rightarrow x^{*} \in K$. Owing to $T: K \rightarrow 2^{\mathbb{R}^{m}}$ is convex compact-valued and continuous. It follows from Remark 4.1 that there exists $z^{*} \in T\left(x^{*}\right)$ such that $z_{k} \rightarrow z^{*}$ and $\lim _{k \rightarrow \infty} T\left(x_{k}\right)=$ $T\left(x^{*}\right)$. By Theorem 3.4(ii), we have

$$
f\left(z^{*}, x^{*}\right) \leqslant \limsup _{k \rightarrow+\infty} f\left(z_{k+1}, x_{k+1}\right) \leqslant 0 .
$$

For each $y \in \mathbb{R}^{m}$, the function $\Phi(\cdot, \cdot, \cdot-y)$ is lsc, we have

$$
\Phi\left(z^{*}, x^{*}, x^{*}-y\right) \leqslant \liminf _{\kappa \rightarrow+\infty} \Phi\left(z_{k+1}, x_{k+1}, x_{k+1}-y\right) \leqslant 0, \quad \forall y \in K .
$$

Moreover, we get, for any $\gamma>\hat{\gamma}=1$,

$$
g_{\gamma}\left(x^{*}, x^{*}-y\right) \leqslant 0, \quad \forall y \in K .
$$

That is, $x^{*} \in \mathcal{S}$. By Corollary 4.1, $\left(x^{*}, z^{*}\right) \in \Theta$. Therefore, the sequence $\left\{\left(x_{k}, z_{k}\right)\right\}$ generated by Algorithm 5.1 converges to some solution of (BVI).

Remark 5.1 It follows from the proof of Theorem 5.1 that Algorithm 5.1 is exact penalty method.

We give an example to show how Algorithm 5.1 can be used for finding a solution of a concrete (BVI).

Example 5.1 Let $K, \psi$ and $\Phi$ be the same as Example 3.4, and for each $x \in K$, $T(x)=\left[x,-10^{-15}\right]$. (BVI) is defined as follows: find $x \in K=\left[-1,-\frac{1}{2}\right]$ such that

$$
\left\langle-z x \mathrm{e}^{z}, x-y\right\rangle \leqslant 0, \quad \forall y \in\left[-1,-\frac{1}{2}\right],
$$

where $z$ solves the following parametric variational inequality: find $z \in T(x)=$ $\left[x,-10^{-15}\right]$ such that

$$
\left\langle\mathrm{e}^{z}, z-v\right\rangle \leqslant 0, \quad \forall v \in\left[x,-10^{-15}\right]
$$


Obviously, $z=x$ is the unique solution of parametric variational inequality (5.9) and so $\left(-\frac{1}{2},-\frac{1}{2}\right)$ is the unique solution of (BVI) (5.8)-(5.9). Solving the (BVI) (5.8)(5.9) by Algorithm 5.1, we should firstly transform (BVI) into one-level variational inequality. For $\gamma>0, x \in\left[-1,-\frac{1}{2}\right]$ and $z \in T(x)=\left[x,-10^{-15}\right]$, calculating the formula of penalty functions $f$ and $g_{\gamma}$ :

$$
f(z, x)=\sup _{v \in\left[x,-10^{-15}\right]}\left\langle\mathrm{e}^{z}, z-v\right\rangle=(z-x) \mathrm{e}^{z},
$$

and so, for each $y \in\left[-1,-\frac{1}{2}\right]$,

$$
g_{\gamma}(x, x-y)=\Phi(z, x, x-y)+\gamma f(z, x)=\mathrm{e}^{z}\left(-x^{2} z+x y z+\gamma z-\gamma x\right) .
$$

It is easy to see that for each $x \in K, g_{\gamma}(x, x-\cdot)$ is strictly monotone. We will use the strict monotonicity of $g_{\gamma}$ to verify the inequality (5.1) of Algorithm 5.1. Next, we illustrate the process of solving (BVI) (5.8)-(5.9) by Algorithm 5.1.

Step 1. Take $\gamma_{k}=2+k$. Choose $x_{0}=-1$ and the tolerance $\varepsilon=10^{-5}$.

Step 2. Verify whether there exists $z_{0} \in T\left(x_{0}\right)=\left[-1,-10^{-15}\right]$ such that (5.1) holds. By the strict monotonicity of $g_{\gamma}$, applying the descent direction method solving the minimization problem:

$$
\min _{y \in K}-g_{\gamma_{0}}\left(x_{0}, x_{0}-y\right) .
$$

We can conclude that $y=-\frac{1}{2}$, which is an optimal solution of (5.10), but

$$
g_{\gamma_{0}}\left(x_{0}, x_{0}-y\right)=\frac{1}{2 \mathrm{e}}>\varepsilon .
$$

So, pick up $z_{0}=-1$ and go to step 3 of Algorithm 5.1.

Step 3. Compute $x_{1} \in K$.

Find $z_{1} \in T\left(x_{1}\right)=\left[x_{1},-10^{-15}\right]$ such that (5.3) holds. Then

$$
f\left(z_{1}, x_{1}\right)=\left(x_{1}-z_{1}\right) \mathrm{e}^{z_{1}} \leqslant \max \left\{0, \frac{1}{\gamma_{0}} f\left(z_{0}, x_{0}\right)\right\}=\frac{1}{2}\left(x_{0}-z_{0}\right) \mathrm{e}^{z_{0}}=0 .
$$

We can obtain $z_{1}=x_{1}$. By (5.4), solving the following minimization problem by descent direction method:

$$
\min _{y \in K}-g_{\gamma_{1}}\left(x_{1}, x_{1}-y\right),
$$

that is,

$$
\min _{y \in K}-\mathrm{e}^{x_{1}}\left(-x_{1}^{3}+x_{1}^{2} y\right) .
$$

Hence $y=-\frac{1}{2}$ and therefore, for each $y \in K$,

$$
g_{\gamma_{1}}\left(x_{1}, x_{1}-y\right) \leqslant-x_{1}^{2} \mathrm{e}^{x_{1}}\left(x_{1}+\frac{1}{2}\right) .
$$


Solving (5.4) is equivalent to find $x_{1} \in\left[-1,-\frac{1}{2}\right]$ such that

$$
-x_{1}^{2} \mathrm{e}^{x_{1}}\left(x_{1}+\frac{1}{2}\right) \leqslant \varepsilon
$$

Again using tangent method, we can obtain a unique solution $x_{1}=-\frac{1}{2}$ such that (5.11) hold. Then $z_{1}=-\frac{1}{2}$. Substitute $z_{1}=-\frac{1}{2}$ and $x_{1}=-\frac{1}{2}$ into (5.1), we have

$$
\Phi\left(z_{1}, x_{1}, x_{1}-y\right)+3 f\left(z_{1}, x_{1}\right)=\mathrm{e}^{-\frac{1}{2}}\left(\frac{1}{8}+\frac{1}{4} y\right) \leqslant \varepsilon, \quad \forall y \in K .
$$

Then stop and output $z_{1}=-\frac{1}{2}$ and $x_{1}=-\frac{1}{2}$. That is, $\left(-\frac{1}{2},-\frac{1}{2}\right)$ is a solution of (BVI) (5.8)-(5.9).

\section{Relationships Among (BVI), (SVI) and (VVI)}

In this section, we shall discuss the relationships among (BVI), system of variational inequalities (SVI) and vector variational inequalities (VVI).

We consider the following (SVI) and (VVI) defined by, respectively,

(SVI): find $(x, z) \in K \times T(x)$ such that

$$
\begin{cases}\Phi(z, x, x-y) \leqslant 0, & \forall y \in K \\ \psi(z, z-v) \leqslant 0, & \forall v \in T(x) .\end{cases}
$$

(VVI): find $(x, z) \in K \times T(x)$ such that

$$
(\Phi(z, x, \cdot), \psi(z, \cdot))((x, z)-(y, v)) \in-\mathbb{R}_{+}^{2}, \quad \forall(y, v) \in K \times T(x),
$$

where $(\Phi(z, x, \cdot), \psi(z, \cdot))((x, z)-(y, v))=(\Phi(z, x, x-y), \psi(z, z-v))$.

It is easy to see that (SVI) and (VVI) are equivalent.

Lemma 6.1 If $(x, z) \in \Theta$, then $(x, z)$ is a solution of $(S V I)(\operatorname{or}(V V I))$.

Proof Assume that $(x, z) \in \Theta$. Then $x \in K$ such that

$$
\Phi(z, x, x-y) \leqslant 0, \quad \forall y \in K, z \in M(x) .
$$

This implies that $(x, z) \in K \times T(x)$ such that (6.1) (or (6.2)) holds. Thus, $(x, z)$ is a solution of (SVI) (or (VVI)).

Example 6.1 Let $\Phi(z, x, x-y)=\langle 2 z+1, x-y\rangle$, and let $K, T$ and $\psi$ be the same as Example 2.1. Then

$$
M(x)= \begin{cases}\{-1\}, & \text { if } x \in\left[-1,-\frac{3}{4}\right), \\ \left\{-\frac{3}{4}\right\}, & \text { if } x \in\left[-\frac{3}{4},-\frac{2}{3}\right), \\ \left\{-\frac{2}{3},-\frac{3}{4}\right\}, & \text { if } x \in\left[-\frac{2}{3},-\frac{1}{2}\right), \\ \left\{-\frac{1}{2},-\frac{2}{3},-\frac{3}{4}\right\}, & \text { if } x=-\frac{1}{2} .\end{cases}
$$


After computation, $\left\{\left(-\frac{1}{2},-\frac{1}{2}\right),\left(-\frac{1}{2},-\frac{2}{3}\right),\left(-\frac{1}{2},-\frac{3}{4}\right)\right\}$ is the solution set of (BVI). Clearly, $\left\{\left(-\frac{1}{2},-\frac{1}{2}\right),\left(-\frac{1}{2},-\frac{2}{3}\right),\left(-\frac{1}{2},-\frac{3}{4}\right)\right\}$ is also the solution set of (SVI) and (VVI).

Generally speaking, the converse of Lemma 6.1 may be not true.

Example 6.2 Let $\Phi(z, x, x-y)=\langle 3 z+2, x-y\rangle$, and let $K, T$ and $\psi$ be the same as Example 2.1. It is easy to verify that $\left\{\left(t,-\frac{2}{3}\right): t \in\left[-\frac{2}{3},-\frac{1}{2}\right)\right\}$ is the solution set of (SVI) and (VVI). But (BVI) has no solution.

\section{Concluding Remarks}

In this paper, we introduce and investigate a general model of bilevel variational inequalities (BVI) with hierarchical nesting structure, which involves two variational inequalities where the constraint region of the upper-level variational inequality problem (called the leader's variational inequality problem) is implicitly determined by the lower-level variational inequality (called the follower's variational inequality) parameterized in the leader's decision variable. (BVI) provides a unity framework to deal with decision processes involving two decision makers with a hierarchical structure. The relationships between (BVI) and existing bilevel problems are presented. Subsequently, the existence of solution to (BVI) and (LSVI) are proved without coercivity. The closedness and compactness of solution sets to (BVI) and (LSVI) are also derived under some suitable conditions. By using the penalty method, we transform (BVI) into one-level variational inequality, and establish the equivalence between (BVI) and the one-level variational inequality. A new iterative algorithm to compute the approximate solutions of (BVI) is suggested and analyzed. The strong convergence of the iterative sequence generated by the proposed algorithm is also established under some mild conditions. Last but not the least, some relationships among (BVI), system of variational inequalities and vector variational inequalities are discussed. These results are new and generalize some recent results in this field.

Throughout this paper, we present the sufficient optimality for (BVI) under the assumptions which ensure the rational reaction set $M(x)$ is a singleton with respect to the current decision $x$ of the upper-level decision maker, i.e., (BVI) (2.1)-(2.2) is studied in the sense of optimism. We say the (BVI) is an optimistic bilevel variational inequalities whenever the rational reaction set $M(x)$ is a singleton with respect to the current decision $x$ of the upper-level decision maker. Otherwise, we say the (BVI) is a pessimistic bilevel variational inequalities. It is worth noting that (LSVI) is a parametric variational inequalities and so, its solution set is a set-valued mapping with respect to the upper-level decision variable. Example 2.1 shows that (BVI) may be not stable, if the rational reaction set $M(x)$ is not a singleton for some fixed $x \in C(X)$. Moreover, (BVI) may exist an optimal solution even though the rational reaction set $M(x)$ is not a singleton for some fixed $x \in C(X)$ (see, Examples 2.2 and 6.1).

For this reason, one shall investigate the behavior of the solution set of (LSVI) such as lower semicontinuity and upper semicontinuity, and study the sufficient and necessary conditions under which (BVI) is stable and solvable when the solution of (LSVI) is not unique. 
Another important topic is how to construct effective and implement algorithms for (BVI) if the rational reaction set $M(x)$ is not unique for some fixed $x \in C(X)$. However, it is hard to obtain exact solutions in many practical problems. We often only derive the approximate solution of (BVI). As we know, any approximating sequence of solutions generated by algorithm is meaningful whenever the problem under consideration is well-posed. For parametric problems, well-posedness is closely related to stability. Therefore, it is also deserved to define several appropriate concepts of well-posedness for (BVI) and give some classes of functions such that (BVI) is well-posed (see, e.g., [1, 30, 31] etc.).

We observe that the upper-level variational inequality (2.1) of (BVI) holds for each solution of the lower-level one. This constraint has a bit harsh. When the rational reaction set $M(x)$ is not a singleton for some fixed $x \in C(X)$, the leader is allowed to select the element in $M(x)$ that satisfies its objectives. In this case, we may introduce and study the following relaxed bilevel variational inequalities: find $x \in K$ such that there exists $z \in S(x)$,

$$
\Phi(z, x, x-y) \leqslant 0, \quad \forall y \in K,
$$

where $S(x)$ is the solution set of the following variational inequalities defined by bifunction with respect to a parameter $x$ : find $z \in T(x)$ such that

$$
\psi(z, z-v) \leqslant 0, \quad \forall v \in T(x) .
$$

It is worthwhile to be consider the existence of solution and algorithms for (BVI) (7.1)-(7.2) from theoretical and practical point of view. These topics will be done in the future.

Acknowledgements The authors wish to thank the anonymous referees and associated editor for their very careful and valuable comments which led to an improved presentation of this manuscript.

\section{References}

[1] Anh, L.Q., Khanh, P.Q., Van, D.T.M.: Well-posedness under relaxed semicontinuity for bilevel equilibrium and optimization problems with equilibrium constraints. J. Optim. Theory Appl. 153, 42-59 (2012)

[2] Anh, P.N., Kim, J.K., Muu, L.D.: An extragradient algorithm for solving bilevel pseudomonotone variational inequalities. J. Glob. Optim. 52, 627-639 (2012)

[3] Aubin, J.P., Ekeland, I.: Applied Nonlinear Analysis. Wiley, New York (1984)

[4] Bank, B., Guddat, J., Klatte, D., Kummer, B., Tammer, K.: Nonlinear Parametric Optimization. Akademie-Verlag, Berlin (1982)

[5] Bard, J.F.: Practical Bilevel Optimization: Algorithm and Applications. Kluwer Academic, Dordrecht (1998)

[6] Berge, C.: Topological Spaces. Oliver and Boyd, London (1963)

[7] Birbil, S.I., Bouza, G., Frenk, J.B.G., Still, G.: Equilibrium constrained optimization problems. Eur. J. Oper. Res. 169, 1108-1127 (2006)

[8] Cervinka, M.: Hierarchical structures in equilibrium problems. Ph.D. Thesis, Department of probability and mathematical statistics, Charles University in Prague and Institute of information theory and automation, academy of sciences of the Czech Republic (2008)

[9] Chadli, O., Mahdioui, H., Yao, J.C.: Bilevel mixed equilibrium problems in Banach spaces: existence and algorithmic aspects. Numer. Algebra Cont. Optim. 1(3), 549-561 (2011)

[10] Chen, G.Y.: Existence of solutions for a vector variational inequality: an extension of the HartmanStampacchia theorem. J. Optim. Theory Appl. 74, 445-456 (1992) 
[11] Chen, J.W., Wan, Z.: Existence of solutions and convergence analysis for a system of quasivariational inclusions in Banach spaces. J. Inequal. Appl. 49, 14 (2011). doi:10.1186/1029-242X2011-49

[12] Chen, G.Y., Huang, X.X., Yang, X.Q.: Vector Optimization: Set-Valued and Variational Analysis. Lecture Notes in Economics and Mathematical Systems, vol. 285, pp. 408-416. Springer, Berlin (2005)

[13] Chen, J.W., Wan, Z., Zou, Y.: Bilevel invex equilibrium problems with applications. Optim. Lett. (2012). doi:10.1007/s11590-012-0588-Z

[14] Chen, J.W., Wan, Z., Cho, Y.J.: Levitin-Polyak well-posedness by perturbations for systems of setvalued vector quasi-equilibrium problems. Math. Methods Oper. Res. 77, 33-64 (2013)

[15] Colson, B., Marcotte, P., Savard, G.: Bilevel programming: a survey. Q. J. Oper. Res. 3, 87-107 (2005)

[16] Crespi, G.P., Ginchev, I., Rocca, M.: Minty variational inequalities, increase-along-rays property and optimization. J. Optim. Theory Appl. 123(3), 479-496 (2004)

[17] Crespi, G.P., Ginchev, I., Rocca, M.: Existence of solutions and star-shapedness in Minty variational inequalities. J. Glob. Optim. 32(4), 485-494 (2005)

[18] Dempe, S.: Foundations of Bilevel Programming. Kluwer Academic, Dordrecht (2002)

[19] Dempe, S.: Annotated bibliography on bilevel programming and mathematical programs with equilibrium constraints. Optimization 52, 333-359 (2003)

[20] Dempe, S., Dutta, J.: Is bilevel programming a special case of a mathematical program with complementarity constraints? Math. Program. 131, 37-48 (2012)

[21] Dempe, S., Kalashnikov, V., Rios-Mercado, R.Z.: Discrete bilevel programming: application to a natural gas cash-out problem. Eur. J. Oper. Res. 166, 469-488 (2005)

[22] Ding, X.P.: Auxiliary principle and algorithm for mixed equilibrium problems and bilevel mixed equilibrium problems in Banach spaces. J. Optim. Theory Appl. 146, 347-357 (2010)

[23] Ding, X.P.: Existence and algorithm of solutions for mixed equilibrium problems and bilevel mixed equilibrium problems in Banach spaces. Acta Math. Sin. Engl. Ser. 28(3), 503-514 (2011)

[24] Ding, X.P.: Bilevel generalized mixed equilibrium problems involving generalized mixed variationallike inequality problems in reflexive Banach spaces. Appl. Math. Mech. 32(11), 1457-1474 (2011)

[25] Ding, X.P.: Existence and iterative algorithm of solutions for a class of bilevel generalized mixed equilibrium problems in Banach spaces. J. Glob. Optim. 53, 525-537 (2012)

[26] Ding, X.P., Liou, Y.C., Yao, J.C.: Existence and algorithms for bilevel generalized mixed equilibrium problems in Banach spaces. J. Glob. Optim. 53, 331-346 (2012)

[27] Dinh, B.V., Muu, L.D.: On penalty and gap function methods for bilevel equilibrium problems. J. Appl. Math. 2011, 1-14 (2011). doi:10.1155/2011/646452

[28] Facchinei, F., Pang, J.S.: Finite Dimensional Variational Inequalities and Complementarity Problems, vols. I and II. Springer, New York (2003)

[29] Fan, K.: A generalization of Tychonoff's fixed point theorem. Math. Ann. 142, 305-310 (1961)

[30] Fang, Y.P., Hu, R.: Parametric well-posedness for variational inequalities defined by bifunctions. Comput. Math. Appl. 53, 1306-1316 (2007)

[31] Fang, Y.P., Hu, R., Huang, N.J.: Well-posedness for equilibrium problems and for optimization problems with equilibrium constraints. Comput. Math. Appl. 55, 89-100 (2008)

[32] Fukushima, M., Pang, J.S.: Convergence of a smoothing continuation method for mathematical programs with complementarity constraints. In: Ill-Posed Variational Problems and Regularization Techniques, vol. 477. Springer, Berlin (1999)

[33] Giannessi, F.: Theorems of alterative, quadratic programs and complementary problems. In: Cottle, R.W., Giannessi, F., Lions, J.C. (eds.) Variational Inequality and Complementary Problems. Wiley, New York (1980)

[34] Giannessi, F.: On Minty variational principle. In: Giannessi, F., Komloski, S., Tapcsack, T. (eds.) New Trends in Mathematical Programming, pp. 93-99. Kluwer Academic, Dordrecht (1998)

[35] Guu, S.M., Li, J.: Vector variational-like inequalities with generalized bifunctions defined on nonconvex sets. Nonlinear Anal. 71(7-8), 2847-2855 (2009)

[36] Huang, N.J., Li, J., Thompson, B.H.: Stability for parametric implicit vector equilibrium problems. Math. Comput. Model. 43, 1267-1274 (2006)

[37] Kassay, G., Kolumban, J., Pales, Z.: On Nash stationary points. Publ. Math. (Debr.) 54, 267-279 (1999)

[38] Kien, B.T.: On the lower semicontinuity of optimal solution sets. Optimization 54, 123-130 (2005)

[39] Li, J., Huang, N.J., Yang, X.Q.: Weak sharp minima for set-valued vector variational inequalities with an application. Eur. J. Oper. Res. 205, 262-272 (2010) 
[40] Lin, L.J.: Mathematical programming with system of equilibrium constraints. J. Glob. Optim. 37, 275-286 (2007)

[41] Lin, L.J.: Existence theorems for bilevel problem with applications to mathematical program with equilibrium constraint and semi-infinite problem. J. Optim. Theory Appl. 137(1), 27-40 (2008)

[42] Luo, Z.Q., Pang, J.S., Ralph, D.: Mathematical Programs with Equilibrium Constraints. Cambridge University Press, Cambridge (1996)

[43] Lv, Y., Chen, Z., Wan, Z.: A neural network approach for solving mathematical programs with equilibrium constraints. Expert Syst. Appl. 38, 231-234 (2011)

[44] Mordukhovich, B.S.: Equilibrium problems with equilibrium constraints via multiobjective optimization. Optim. Methods Softw. 19, 479-492 (2004)

[45] Mordukhovich, B.S., Outrata, J.V., Cervinka, M.: Equilibrium problems with complementarity constraints: case study with applications to oligopolistic markets. Optimization 56(4), 479-494 (2007)

[46] Moudafi, A.: Proximal methods for a class of bilevel monotone equilibrium problems. J. Glob. Optim. 47, 287-292 (2010)

[47] Nagurney, A., Yu, M.: Sustainable fashion supply chain management under oligopolistic competition and brand differentiation. Int. J. Prod. Econ. 135(2), 532-540 (2012)

[48] Outrata, J.V.: A note on a class of equilibrium problems with equilibrium constraints. Kybernetika 40, 585-594 (2004)

[49] Robinson, S.M.: Solution continuity affine variational inequalities. SIAM J. Optim. 18, 1046-1060 (2007)

[50] Scheel, H., Scholtes, S.: Mathematical programs with complementarity constraints: stationarity, optimality and sensitivity. Math. Oper. Res. 25(1), 1-22 (2000)

[51] Stampacchia, G.: Forms bilineaires coercivities sur les ensembles convexes. C. R. Math. Acad. Sci. Paris 258, 4413-4416 (1964)

[52] Su, C.L.: Equilibrium problems with equilibrium constraints: stationarities, algorithms, and applications. Ph.D. Thesis, Department of Management Science and Engineering, Stanford University, Stanford, CA (2005)

[53] Vicente, L., Calamai, P.H.: Bilevel and multibilevel programming: a bibliography review. J. Glob. Optim. 5, 291-305 (1994)

[54] Wan, Z., Chen, J.W., Sun, H., Yuan, L.: A new system of generalized mixed quasivariational inclusions with relaxed cocoercive operators and applications. J. Appl. Math. 2011, 1-26 (2011). doi:10.1155/2011/961038

[55] Wong, M.M.: Lower semicontinuity of the solution map to a parametric vector variational inequality. J. Glob. Optim. 46, 435-446 (2010)

[56] Xu, H.F., Ye, J.J.: Necessary optimality conditions for two-stage stochastic programs with equilibrium constraints. SIAM J. Optim. 20(4), 1685-1715 (2010)

[57] Xu, M.H., Li, M., Yang, C.C.: Neural networks for a class of bi-level variational inequalities. J. Glob. Optim. 44, 535-552 (2009)

[58] Yang, X.Q., Yao, J.C.: Gap functions and existence of solutions to set-valued vector variational inequalities. J. Optim. Theory Appl. 115, 407-417 (2002)

[59] Ye, J.J.: Necessary and sufficient optimality conditions for mathematical programs with equilibrium constraints. J. Math. Anal. Appl. 307, 305-369 (2005)

[60] Ye, J.J., Ye, X.Y.: Necessary optimality conditions for optimization problems with variational inequality constraints. Math. Oper. Res. 22, 977-997 (1997)

[61] Yuan, G.X.-Z., Isac, G., Tan, K., Yu, J.: The study of minimax inequalities, abstract economics and applications to variational inequalities and Nash equilibria. Acta Appl. Math. 54, 135-166 (1998) 